ISSN 1112-9867

Available online at

http://www.jfas.info

\title{
CALCULATION OF IONIZATION RATE COEFFICIENTS OF HELIUMOIDE IRON
}

\author{
S. Dilmi* and E. Guedda \\ VTRS et Institut des Sciences et technologie, Centre universitaire d'El-Oued., \\ BP.789, El-Oued 39 000, ALGERIE
}

Received: 25 November 2012 / Accepted: 02 December 2012 / Published online: 30 June 2012

\begin{abstract}
The atomic data of the various atomic processes are essential for the radiative collisional models describing plasmas. In this work we have our computation results of the coefficients of ionization rate of iron héliumoîde. These types of ions can exist in all the types of plasmas, amongst other things plasmas laser. We use a simple numerical method to generate the ionization rates starting from the cross sections. These last are obtained by code FAC (Flexible Atomic Code). Our computation results will be compared with those published.
\end{abstract}

Key words: Atomic data, radiative collisional Models, Ionization rate, cross Sections of ionization.

\section{INTRODUCTION}

Plusieurs problèmes liés à l'étude des plasmas de laboratoire, d'astrophysique et de fusion thermonucléaire contrôlée nécessitent la connaissance au préalable de la structure atomique comme les énergies et longueurs d'onde des différents niveaux et les sections efficaces et les coefficients de taux des processus élémentaires dans les plasmas. Cependant, le calcul direct de ces données devient impossible pour les atomes à plusieurs électrons et aussi pour les grands nombres quantiques.

Author Correspondence, e-mail: sam1dilmi@yahoo.fr

ICID: 1020710 
Dans ce cas on fait recours aux programmes et codes de calcul. Malgré le nombre important des codes existant pour le traitement des données atomiques, ils sont tous en compétition pour donner des résultats proches de l'expérimental. Le code FAC (Flexible Atomic Code) [1] a été choisi pour calculer les sections efficaces d'ionisation du fer héliumoïde. Les taux d'ionisation du fer héliumoïde seront générés par un calcul numérique.

\section{ASPECT THEORIQUE}

Dans le processus d'ionisation par l'électron d'impact, un atome ou ion peut heurter un électron, il s'ionise en cédant aussi un électron:

$$
(\mathrm{Z}, \mathrm{N})+\mathrm{e} \Leftrightarrow(\mathrm{Z}, \mathrm{N}-1)+\mathrm{e}+\mathrm{e}
$$

Où $(\mathrm{Z}, \mathrm{N})$ représente un ion de charge $\mathrm{Z}$ possédant $\mathrm{N}$ électrons.

Les sections efficaces d'ionisation par électron d'impact peuvent être évaluées théoriquement comme fonction du rapport de l'énergie de l'électron d'impact $\boldsymbol{\varepsilon}$ et le potentiel d'ionisation $\chi Z$ de l'ion de charge Z.

\section{Méthode d'évaluation des sections efficaces}

Plusieurs équations sont présentées par Kato et al. [2] pour le calcul des sections efficaces et coefficients de taux d'ionisation. Parmi eux on peut citer l'équation de Lotz, Arnaud et al. [3] et celui du groupe de Belfast [4-5]. L'équation de Lotz a été largement utilisée pour le calcul des sections efficaces et les coefficients de taux d'ionisation [6-7]. Lotz déduit la section efficace d'ionisation en se basant sur des données expérimentales pour les ions faiblement ionisés avec des faibles charges $\mathrm{Z}$ :

$$
\sigma=\sum_{j=1}^{N a s} a_{j} \zeta_{j} \frac{l n u_{j}}{u_{j} \chi_{j}^{2}}\left\{1-b_{j} \exp \left[-c_{j}\left(u_{j}-\mathbf{1}\right)\right]\right\}
$$

Où $u_{j}=\frac{\varepsilon}{\chi_{j}}$ est l'énergie de liaison d'un électron dans la $j^{e m \varepsilon}$ sous couche, $\zeta_{j}$ est le nombre des électrons équivalents dans la sou couche $\mathrm{j}$ alors que $a_{j}, b_{j}$ et $c_{j}$ sont des constantes qui peuvent être ajustées à partir des données expérimentales et des lois d'échelle. 
Le coefficient de taux d'ionisation $\left(\mathrm{cm}^{3} \cdot \mathrm{s}^{-1}\right)$ est obtenu par intégration de la section efficace sur une distribution maxwellienne:

$$
s=6.710^{7} \sum_{j=1}^{N} \frac{a_{j} \zeta_{j}}{T_{\theta}^{\frac{3}{2}}}\left\{\frac{1}{\frac{\chi_{j}}{T_{\theta}}} \int_{\frac{x_{j}}{T_{\theta}}} \frac{\exp (-x)}{x} d x-\frac{b_{j} \exp c_{j}}{\frac{\chi_{j}}{T_{\theta}}+c_{j}} \int_{\frac{\chi_{j}}{T_{e}}+c_{j}}^{\infty} \frac{\exp (-\square)}{y} d y\right\}
$$

\section{Calcul des coefficients de taux d'ionisation du fer héliumoïde}

Le coefficient de taux d'ionisation $\tau$ est donné en fonction de la section efficace d'ionisation $\sigma(E)$ par l'équation:

$$
\begin{aligned}
& \tau=\int v \sigma(E) f(E) d E \quad\left(\mathrm{~cm}^{3} \mathrm{~s}^{-1}\right) \\
& v=\sqrt{\frac{2 E}{M}} \quad ; \quad f(E) d E=\frac{2 \sqrt{E} e^{-\frac{E}{T}}}{T^{3 / 2} \sqrt{\pi}} d E
\end{aligned}
$$

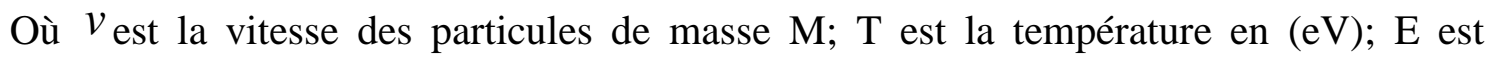
l'énergie 'Threshold' d'ionisation de lithium héliumoïde; et $f(E)$ est la distribution maxwellienne pour l'énergie.

\section{Méthode de calcul}

Les sections efficaces d'ionisation du fer héliumoïde sont obtenues par le code FAC [1]. Les coefficients de taux d'ionisation seront calculés suivant l'équation (4). Le calcul numérique de l'intégral présenté par l'équation (4) est basé sur la méthode de trapèze.

\section{RESULTATS ET DISCUTIONS}

Les résultats de calcul des taux d'ionisation du fer héliumoïde obtenus seront reportés sur la figure 1. Ces résultats seront comparés à ceux publiés par Kato et al. [2]. En général, une bonne concordance a été remarquée sur les deux courbes de la figure 1. Cependant le tableau 1 montre les erreurs relatives entre les taux de Kato et al ( $\left.\tau_{\text {Kato }}\right)$ et ceux du présent travail $(\tau)$. Les erreurs calculées sont considérables dans la gamme 5 . $10^{3} \mathrm{eV}-4.10^{4} \mathrm{eV}$, alors qu'elles sont trop faibles autour de $1500 \mathrm{eV}$. 
Tableau 1. Les erreurs relatives des taux d'ionisation.

\begin{tabular}{|c|c|c|c|c|}
\hline $\mathrm{T}(\mathrm{eV})$ & $\tau$ & $\tau_{\text {Kato }}$ & $\tau_{\text {Belfast }}$ & $\tau / \tau$ \\
\hline 1500 & $8.2352 \mathrm{E}-15$ & $8.2352 \mathrm{E}-15$ & & 0.00 \\
\hline 6100 & $1.5257 \mathrm{E}-12$ & $8.9575 \mathrm{E}-13$ & $5.1379 \mathrm{E}-13$ & $0.41-0.66$ \\
\hline 40100 & $4.2086 \mathrm{E}-12$ & $4.2086 \mathrm{E}-12$ & $2.3562 \mathrm{E}-12$ & $0.00-0.43$ \\
\hline
\end{tabular}

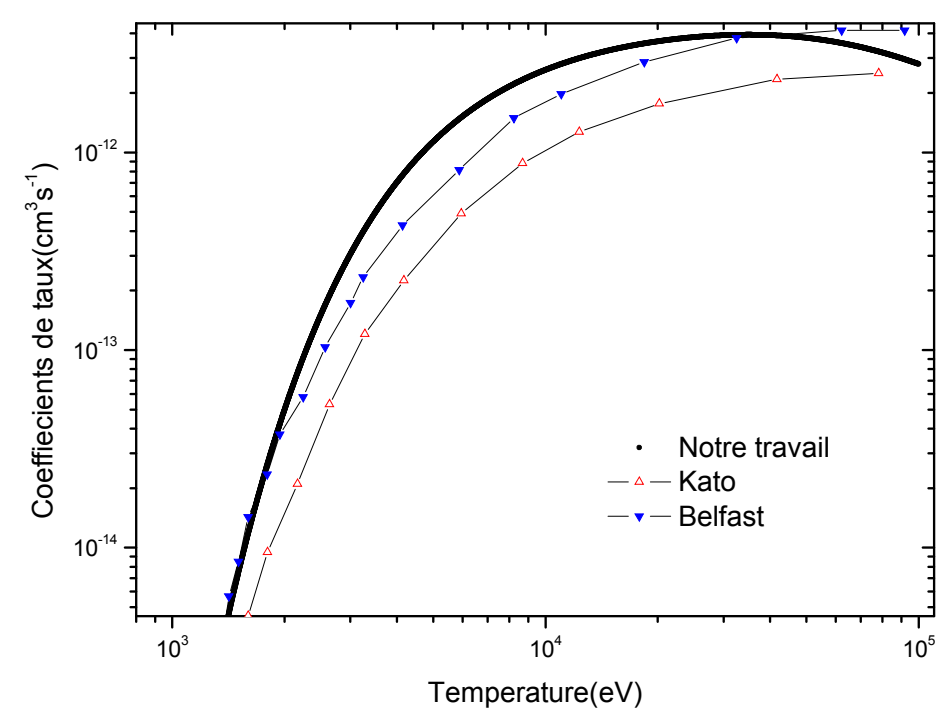

Fig.1. Coefficients de taux d'ionisations pour le fer héliumoïde

\section{CONCLUSION}

Les coefficients de taux d'ionisation du fer héliumoïde ont été calculés à partir des sections efficaces. Ces dernières ont été obtenues par le code FAC. Les résultats obtenus sont, généralement, en bonne concordance avec les résultats publiés.

\section{REFERENCES}

[1] Gu M. F. FAC 1.0.7, http://kipac-tree.stanford.edu/fac/

[2] Kato T. et al. NIFS-Data-Series. 1991, 14.

[3] Arnaud M. and Rothenflug R. Astron. Astrophys. Supp. Ser. 1985, 60, 425.

[4] Bell K. L. et al. J. Phys. Chem. Ref. Data. 1983, 12, 891.

[5] Lennon M. A. et al. J. Phys. Chem. Ref. Data. 1988, 17,1285. 
[6] Shevelko V. P. and Vainshtein L. A. 1993, Atomic Physics for Hot Plasmas, IOP Publishing, london.

[7] Younger S. M. JQSRT. 1981, 26, 329.

\section{CALCUL DES COEFFICIENTS DE TAUX D'IONISATION DU FER HELIUMOIDE}

\section{RESUME}

Les données atomiques des différents processus atomiques sont indispensables pour les modèles collisionnels radiatifs décrivant les plasmas. Dans ce travail nous présentons nos résultats de calcul des coefficients de taux d'ionisation du fer héliumoîde. Ces types d'ions peuvent exister dans tous les types de plasmas, entre autre les plasmas laser. Nous utilisons une méthode numérique simple pour générer les taux d'ionisation à partir des sections efficaces. Ces dernières sont obtenues par le code FAC (Flexible Atomic Code). Nos résultats de calcul seront comparés à ceux publiés.

Mots clés: Données atomiques, Modèles collisionnels radiatifs, Taux d'ionisation, Sections efficaces d'ionisation.

\section{How to cite this article}

Dilmi S and Guedda E. Calculation of ionization rate coefficients of heliumoide iron. $\mathbf{J}$ Fundam Appl Sci. 2012, 4(1), 59-63. 